\title{
Influence of fifth-order nonlinearities on the statistical fluctuations in emission intensities in a photonic open-cavity complex system
}

\author{
Iván R. R. González $\odot,{ }^{1}$ Ernesto P. Raposo,${ }^{1,2,3}$ Sandra J. Carreño $\odot,{ }^{4}$ Antônio M. S. Macêdo $\odot,{ }^{1}$ Melissa Maldonado $\odot,{ }^{5}$ \\ Leonardo de S. Menezes $\odot,{ }^{5}$ Anderson S. L. Gomes $\odot,{ }^{5}$ and Cid B. de Araújo $\oplus^{5}$ \\ ${ }^{1}$ Laboratório de Física Teórica e Computacional, Departamento de Física, Universidade Federal de Pernambuco, \\ Recife-PE 50670-901, Brazil \\ ${ }^{2}$ Centre d'Estudis Avançats de Blanes-CEAB-CSIC, Girona, 17300, Spain \\ ${ }^{3}$ Centre for Ecological Research and Forestry Applications-CREAF, Universitat Autònoma de Barcelona, 08193, Cerdanyola del Vallès, Spain \\ ${ }^{4}$ Departamento de Eletrônica e Sistemas, Universidade Federal de Pernambuco, Recife-PE 50670-901, Brazil \\ ${ }^{5}$ Departamento de Física, Universidade Federal de Pernambuco, Recife-PE 50670-901, Brazil
}

(Received 8 June 2020; accepted 23 November 2020; published 11 December 2020)

\begin{abstract}
High-order nonlinear optical effects have become an important feature of photonic systems, both ordered and disordered. In this work, a very large and robust set of experimental data obtained from the emission spectra of a trivalent neodymium ion-based random laser, whose action mechanism relies upon gain and disorder, was characterized in detail. Using an effective model, it was possible to describe how the optical nonlinearities of the disordered gain medium affect the statistical behavior of the intensity fluctuations of the random laser for excitations close to and well above the laser threshold. A theoretical framework is presented and, in particular, in the regime well above threshold a nice fit to the experimental data is obtained with a distribution that incorporates nonlinearities up to fifth order, the Izrailev distribution.
\end{abstract}

DOI: 10.1103/PhysRevA.102.063515

\section{INTRODUCTION}

Random lasers (RLs) are fascinating open-cavity optical sources, with several applications in photonics and complex systems [1-10]. This type of device generates laser emission with a cavity-free configuration, where a gain medium amplifies the light emitted by excited atoms, ions, or molecules, and a disordered medium enhances the phenomenon of stimulated emission by providing multiple light scattering. After the experimental observation of Lawandy and coworkers [1], several research groups have explored ingenious types of random lasing architectures. A compendium incorporating the newest ideas and the most up-to-date features can be found in Refs. [2-7], some of which have generated important contributions from science to society. For instance, Polson et al. [8,9] and Wang et al. [10] have shown the feasibility of diagnosing cancerous human tissue using RL devices.

Due to the small lasing threshold and large fluorescence efficiency of rare-earth ions hosted in dielectric structures $[11,12]$, RLs based on trivalent neodymium $\left(\mathrm{Nd}^{3+}\right)$ crystalline powders have become very interesting systems for applications and as test beds of new RL schemes and physical mechanisms [2,13-19].

Despite the outstanding development in RL technology in the past years, the fundamental physics underlying the random fluctuations of the spectrally resolved emission intensity, given the same initial experimental conditions of the excitation beam, remains an open question, which requires full understanding for allowing a greater application palette for such a kind of optical source. A valid approach may be to assume that the random fluctuations are an intrinsic feature of trivalent rare-earth-ions-based RLs [19]. In this scenario, statistical physics methods can provide alternatives to characterize the emission intensities of RL systems.

In addition, the nonlinear optical effects associated with RLs based on $\mathrm{Nd}^{3+}$ crystalline powders include, but are not limited to, Stokes and anti-Stokes frequency conversion $[17,18]$. It is thus a great challenge to provide a detailed forecast about the influence of these effects on the RL emission intensity as a function of the pumping power. In this sense, studies of the random fluctuations of the emission intensity in RL systems have so far considered up to the third-order nonlinearity of the gain medium [17-29].

In this work, an effective model is proposed that considers effects of higher-order nonlinearities on the probability density function (PDF) of RL emission intensities. We present in Sec. II results of a large set of experimental data $\left(10^{5} \mathrm{RL}\right.$ emission spectra) obtained from a $\mathrm{Nd}^{3+}$ crystalline powder based RL system. The theoretical analysis of the results follows in Secs. III and IV. In particular, it is shown in the regime well above threshold that a nice fit to the experimental data is obtained with the Izrailev distribution, which incorporates nonlinearities up to fifth order. At last, conclusions and final remarks are left for Sec. V.

\section{EXPERIMENTAL DETAILS}

The experiments were performed using a crystalline powder of $\mathrm{Nd}_{0.8} \mathrm{Y}_{0.2} \mathrm{Al}_{3}\left(\mathrm{BO}_{3}\right)_{4}$ (NdYAB). The sample was 
(a)

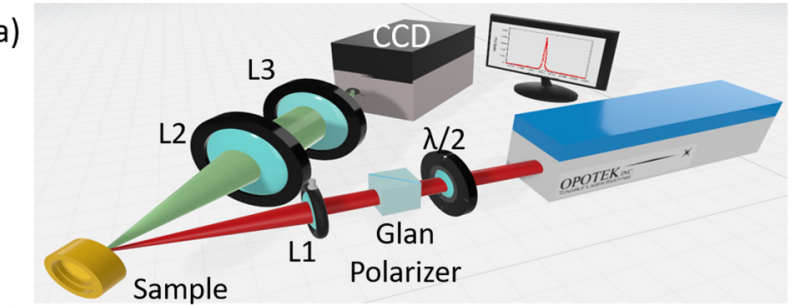

(b)

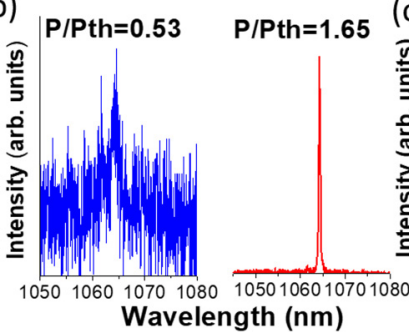

(c)

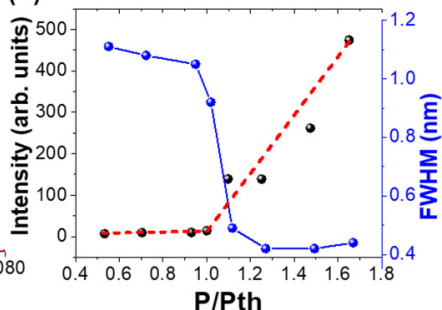

FIG. 1. Characterization of the $\mathrm{Nd}^{3+}$-based random laser (RL). (a) Experimental setup. (b) Emission spectrum of the RL below [blue (left)] and above [red (right)] the laser threshold. (c) Intensity (black circles) and FWHM (blue circles) as a function of the normalized power with respect to the threshold $P / P_{t h}$. The time for acquiring each RL emission spectrum (sampling time) was $100 \mathrm{~ms}$.

prepared by the polymeric precursor method (for further details, see $[12,18])$, and the NdYAB nanocrystals sizes range from 20 to $600 \mathrm{~nm}$.

A scheme of the experimental setup is presented in Fig. 1(a). The excitation source was an optical parametric oscillator (OPO) operating at $808 \mathrm{~nm}(10-\mathrm{Hz}$ repetition rate, 5-ns pulse width). The incident energy was controlled by a half-wave plate and a polarizer, and the beam was focused by a $15-\mathrm{cm}$ focal length lens at $45^{\circ}$ to the surface of the powder. The emitted light was collected at the normal direction to the sample surface by a set of lenses in a microscope configuration, and sent to a spectrometer coupled to a CCD camera with spectral resolution of $0.1 \mathrm{~nm}$.

According to the $\mathrm{Nd}^{3+}$ energy levels in $\mathrm{YAB}$, by exciting the transition ${ }^{4} I_{9 / 2} \rightarrow{ }^{4} F_{5 / 2}$ at $808 \mathrm{~nm}$ one obtains RL emission at $1064 \mathrm{~nm}\left(\mathrm{Nd}^{3+}\right.$ transition $\left.{ }^{4} F_{3 / 2} \rightarrow{ }^{4} I_{11 / 2}\right)$ [18]. Figure 1(b) shows the corresponding emission spectra for two different excitation energies, one below the RL threshold (excitation power normalized by the threshold value $P / P_{t h}=$ 0.53 , left), where the spontaneous emission dominates, and the other above threshold $\left(P / P_{t h}=1.65\right.$, right $)$, where the narrow RL spectral emission is seen. Figure 1(c) shows the RL emission intensity at $1064 \mathrm{~nm}$ and the full width at halfmaximum (FWHM) as a function of $P / P_{t h}$. The abrupt change in the slope of the lines is due to the transition from the spontaneous emission to the RL regime. The RL energy threshold is $0.32 \mathrm{~mJ}$, corresponding to a power of $64 \mathrm{~kW}$.

To perform the statistical analysis of emitted intensities we acquired a large series of $10^{5}$ emission spectra for an excitation power slightly above the RL threshold $\left(P / P_{t h}=1.03\right)$ and the other well above $\left(P / P_{t h}=3.9\right)$. The acquisition time of each spectrum was $100 \mathrm{~ms}$.

In the next sections, a theoretical model that takes into account the third- and fifth-order nonlinear susceptibilities to describe the probability density of emission intensities is presented and discussed.

\section{STOCHASTIC MODEL AND EFFECTIVE THIRD-ORDER SUSCEPTIBILITY CONTRIBUTION}

It is well known that an intense electromagnetic wave traveling through a nonlinear medium with refractive index $n(\mathbf{r})$ generates a material response modeled through Maxwell's equations by

$$
\begin{aligned}
& \nabla \times \mathbf{H}(\mathbf{r}, t)=\epsilon_{0} n^{2}(\mathbf{r}) \partial_{t} \mathbf{E}(\mathbf{r}, t)+\partial_{t} \mathbf{P}_{N L}(\mathbf{r}, t, \mathbf{E}), \\
& \nabla \times \mathbf{E}(\mathbf{r}, t)=-\mu_{0} \partial_{t} \mathbf{H}(\mathbf{r}, t),
\end{aligned}
$$

in the presence of a nonlinear polarization $\mathbf{P}_{\mathrm{NL}}$ [27], whose $i$ th component is generally given by

$$
\begin{aligned}
P_{\mathrm{NL}, i}= & \epsilon_{0}\left(\chi_{i j}^{(1)} E_{j}+\chi_{i j k}^{(2)} E_{j} E_{k}+\chi_{i j k l}^{(3)} E_{j} E_{k} E_{l}\right. \\
& \left.+\chi_{i j k l m}^{(4)} E_{j} E_{k} E_{l} E_{m}+\chi_{i j k l m n}^{(5)} E_{j} E_{k} E_{l} E_{m} E_{n}+\cdots\right)
\end{aligned}
$$

In the expression above, $\chi^{(f)}$ represents the effective nonlinear susceptibility tensor of rank $f+1$ (the term effective is used here to recognize pure $f+1$ nonlinear processes as well as eventual cascade processes). In the case of the $\mathrm{Nd}^{3+}$-based RL, the refractive index $n(\mathbf{r})$ displays a random spatial profile due to the disordered configuration of the nanocrystals in the sample.

The electric field $\mathbf{E}(\mathbf{r}, t)$ can be written in terms of the eigenvalues $\omega_{m}$ and eigenvectors $\mathbf{E}_{m}(\mathbf{r})$ associated with the linear solutions in the form $[28,29]$

$$
\mathbf{E}(\mathbf{r}, t)=\sum_{m} \sqrt{\omega_{m}} a_{m}(t) \mathbf{E}_{m}(\mathbf{r}) \exp \left(-i \omega_{m} t\right)
$$

Below the approximation of slow amplitude modes is considered, in which the phase dynamics of $a_{m}(t)=A_{m}(t) \exp \left[i \phi_{m}(t)\right]$ evolves much faster than its amplitude [28-31]. In this approach, the temporal evolution of the optical modes can be written in terms of the nonlinear polarization and nonlinear susceptibilities. This can be implemented by considering random couplings (denoted by $g_{s p}^{(2)}, g_{s p q r}^{(4)}$, etc.) that incorporate the disordered feature of the RL system with spatially random refractive index $n(\mathbf{r})$. The Langevin equation for the time evolution of the complex amplitudes $a_{m}(t)$ is given by $[28,29]$

$$
\frac{d a_{m}}{d t}=-\frac{\partial \Theta^{(f)}}{\partial a_{m}^{*}}+\eta_{m}(t)
$$

where $\eta_{m}(t)$ denotes a Gaussian white noise with null average and second-order correlation function $\left\langle\eta_{m}(t) \eta_{m^{\prime}}\left(t^{\prime}\right)\right\rangle=$ $2 Q \delta_{m, m^{\prime}} \delta\left(t-t^{\prime}\right)$, with $Q$ as the noise strength. We have considered a sum of additive and multiplicative noises, though only the latter contributes effectively to the model upon averaging out the rapidly varying phases in the slow amplitude modes approach [29]. Several sources can be associated with multiplicative noise processes, including the coupling of the electromagnetic field with stochastic fluctuations of the external pump source, the finite lifetime of photons in the active medium, inversion fluctuations, and polarization fluctuations [32]. Furthermore, all nonlinear effects are included in $\Theta^{(f)}$. The use of the superscript $f$ in $\Theta^{(f)}$ is a reminder of the tensor rank $f+1$ that limits the expansion (2). In other words, $\Theta^{(f)}$ represents the interaction between the electric field and the 
nonlinear polarization expanded in terms of nonlinear susceptibilities up to $\chi^{(f)}$.

This approach was applied [28-31] to connect the phenomenon of replica symmetry breaking (RSB) in photonic systems with their third-order susceptibility. Moreover, this effective model can be also used to find the stationary distribution of emission intensities in disordered systems such as RLs. Recently, this was shown [19,20] by taking into account the effects of the third-order susceptibility, for which $\Theta^{(3)}\left(\chi^{(1)}, \chi^{(3)}\right)=\sum_{s p} g_{s p}^{(2)} a_{s} a_{p}^{*}+$ $\frac{1}{4} \sum_{s p q r} g_{s p q r}^{(4)} a_{s} a_{p} a_{q}^{*} a_{r}^{*}$, with the coupling tensor $g^{(4)}$ related to $\chi^{(3)}$. The Fokker-Planck equation for the intensity $I_{m}=$ $c_{m}\left|a_{m}\right|^{2}$ that follows from Eq. (4) has stationary PDF solution with general form

$$
P\left(I_{m} ; k_{m}, b_{m}\right)=\mathcal{D}_{m} I_{m}^{k_{m}-1} \exp \left(-b_{m} I_{m}\right),
$$

where $\mathcal{D}_{m}$ is the normalization constant, the parameters $k_{m}$ and $b_{m}$ depend on the strength $Q$ of the multiplicative noise, and the couplings $g^{(2)}$ and $g^{(4)}$ are associated with the disordered active medium through

$$
k_{m}=\frac{1}{2 Q}\left(\gamma_{m}-\alpha_{m}-\sum_{r \neq m} \operatorname{Re}\left\{g_{m r m r}^{(4)}+g_{m r r m}^{(4)}\right\} \frac{I_{r}}{2 c_{r}}\right)-1,
$$

and $b_{m}=\operatorname{Re}\left\{g_{m m m m}^{(4)}\right\} /\left(4 Q c_{m}\right)$, in which $\gamma_{m}$ and $\alpha_{m}$ are related to $\operatorname{Re}\left\{g_{m m}^{(2)}\right\}$ and represent, respectively, the amplification (gain) and radiation loss, whereas $g_{m r m r}^{(4)}$ and $g_{m r m}^{(4)}$ are fourthorder couplings between modes $m$ and $r$. Depending on the values of these parameters the PDF (5) assumes the form of a gamma distribution or an exponentially attenuated Lévy-type power-law distribution. For example, if $k_{m}>0$ and $b_{m}>0$, then Eq. (5) corresponds to the gamma distribution. On the other hand, if $k_{m}<0$ and $b_{m}>0$, then Eq. (5) can be conveniently expressed as

$$
P\left(I_{m} ; \mu_{m}, b_{m}\right)=\frac{\mathcal{D}_{m}}{I_{m}^{\mu_{m}}} \exp \left(-b_{m} I_{m}\right),
$$

with $\mu_{m}=1+\left|k_{m}\right|$, which takes the form of a PDF with power-law decay that is exponentially attenuated. We also comment that due to the central limit theorem (CLT) the sum over modes in Eq. (6) leads to small shot-to-shot fluctuations of $k_{m}$ in the regime with many modes. The presence of multiplicative noise generally modifies the mathematical form of the intensity distribution, which would be a Gaussian with only additive noise taken into account [33].

Remarkably, the statistical properties of stochastic phenomena described by distributions (5) and (7) are quite distinct. On the one hand, the gamma distribution (5) is governed by the CLT, showing relatively weak (Gaussian-type) fluctuations [34]. In the photonic RL context, intensity fluctuations of this sort are characteristic of spontaneous emission events below the lasing threshold or the self-averaged Gaussian RL regime well above threshold, in which the gain is shared among a large number of strongly coupled resonance modes [21-26].

In contrast, the heavy-tailed PDF (7) with $b_{m} \rightarrow 0$ corresponds to the large- $I_{m}$ power-law behavior of the Lévy $\alpha$-stable distribution with stability index $\alpha=\mu_{m}-1$ for $\mu_{m} \in(1,3]$, which is given by [34]

$$
P\left(I_{m} ; \alpha, \beta, c, v\right)=\frac{1}{2 \pi} \int_{-\infty}^{\infty} d k \bar{P}(k ; \alpha, \beta, c, v) \exp \left(-i k I_{m}\right),
$$

with characteristic function

$$
\bar{P}(k ; \alpha, \beta, c, v)=\exp \left\{-|c k|^{\alpha}[1-i \beta \operatorname{sgn}(k) \Phi]+i k v\right\} .
$$

Above the stability Lévy index ranges in the interval $\alpha \in$ $(0,2]$. The limit value $\alpha=2$ (i.e., $\mu_{m}=3$ ) corresponds to the Gaussian distribution and CLT [34], whereas values $\mu_{m}>3$ also display $\alpha=2$ Gaussian statistics [35]. The other parameters are $\beta \in[-1,1]$, which describes the skewness of the distribution, the location parameter $v \in(-\infty, \infty)$, the scale parameter $c \in(0, \infty)$, and $\Phi=\tan (\pi \alpha / 2)$ if $\alpha \neq 1$, whereas $\Phi=-(2 / \pi) \ln |k|$ if $\alpha=1$.

In RL systems, once a photon is spontaneously emitted in the disordered active medium the exponential distribution of its path length combined with the multiple random scatterings and stimulated emissions before exiting the material lead [36,37] to a PDF similar to Eq. (7). As many of such photons contribute to the emitted intensity, the PDF (7) converges to the Lévy distribution (8) at least for spectrum acquisition times not too large [21]. In fact, for arbitrarily large number of photons and acquisition times, a crossover from the Lévy to the Gaussian distribution of intensities takes place [21,38-40], as stated by the CLT due to its large but finite second moment. In this work, the strong intensity fluctuations observed near the threshold and the good fit of the high-intensity data to the Lévy distribution (8) (see below) indicate that such crossover has not been reached in our experiment. Indeed, in RL systems with similar acquisition times $[18,19]$ Lévy distributions have been successfully applied to describe the strong intensity fluctuations in the narrow intensity peaks of the RL regime close to the threshold.

In the last two decades, several papers [17-29,36,37,4147] have established the presence of Lévy-type fluctuations near the threshold as a hallmark of RL systems. The mechanism underlying this behavior stems from the presence of optical noise and disorder [19,20,36,37]. In particular, as the excitation power $P$ is raised $k_{m}$ in (5) decreases and $\mu_{m}$ in (7) increases $[19,20]$, so that a phase diagram for the statistical properties of the intensity fluctuations can be outlined $[19,20]$, with a Gaussian regime for $k_{m}>0$ and $P / P_{t h}<1$ that crosses over to the Lévy-type statistics with $1<\mu_{m}<3$ slightly above the RL threshold, and finally to a second (self-averaged) Gaussian regime [21-26] with $\mu_{m} \geqslant 3$ in the RL phase well above threshold.

From the discussion above, we have employed a statistical mixture of a gamma and a Lévy distribution $P\left(I_{m}\right)=$ $p P\left(I_{m} ; k^{(1)}, b^{(1)}\right)+(1-p) P\left(I_{m} ; \alpha^{(2)}, \beta^{(2)}, c^{(2)}, v^{(2)}\right), \quad$ with $0 \leqslant p \leqslant 1$, to the analysis of the histogram of emission intensities in the RL regime close to the threshold. To do this, we considered the data of $10^{5}$ emission spectra for the normalized excitation power $P / P_{t h}=1.03$ and generated a large series with the set of emitted intensities at $1064 \mathrm{~nm}$ (at or very near the spectrum maximum). Below, we denote simply by $I$ the intensity at $1064 \mathrm{~nm}$ of each spectrum.

The intensity of the pump laser presents shot-to-shot fluctuations [48], making the response of the RL close to the 

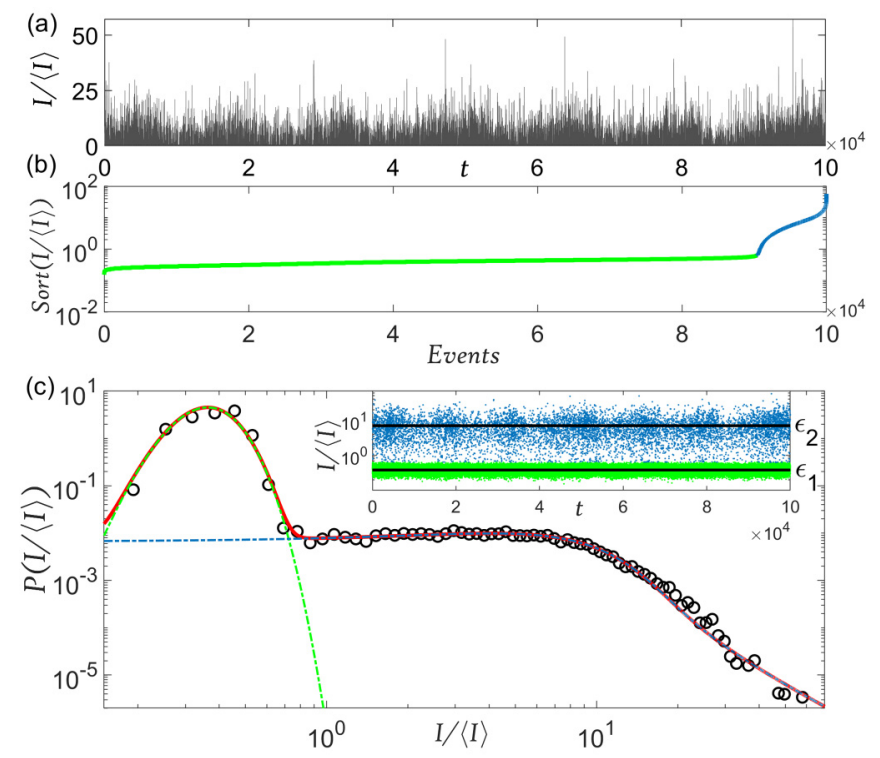

FIG. 2. (a) Long time series of emission intensities $I(t)$ at or very near the maximum of each spectrum (normalized by the mean $\langle I\rangle)$ for an excitation power close to the RL threshold $P / P_{t h}=1.03$. (b) Sorted values by crescent intensity of the series shown in (a), with the statistical weight $p=0.904$ determined from the change of pattern in the intensity values corresponding to the amplified spontaneous (green) and RL (blue) emission regimes. (c) Log-log plot of the PDF $P(I /\langle I\rangle)$ (circles) displaying a nice fit (solid red line) to the statistical mixture of a gamma (dashed green) and a Lévy (dashed blue) distribution $P(I)=p P\left(I, k^{(1)}=22.0, b^{(1)}=57.9\right)+$ $(1-p) P\left(I, \alpha^{(2)}=1.8, \beta^{(2)}=1.0, c^{(2)}=3.4, v^{(2)}=4.2\right)$. From the fitting parameters we determine the mean of the gamma distribution $k^{(1)} / b^{(1)}=0.38$, which is physically consistent with the experimental data in the low-intensity regime. The location parameter $v^{(2)}=4.2$ is also compatible with the maximum of the high-intensity regime. The inset of (c) shows the decomposition of the time series in (a) into events of spontaneous (green) and stimulated (blue) emissions with intensity fluctuations around the means (black horizontal lines).

threshold to vary as well, from amplified spontaneous emission events to lasing peaks. The statistical mixture exhibited by the intensity time series $I(t)$ normalized by the mean $\langle I\rangle$, shown in Fig. 2(a), is a consequence of this effect, which becomes apparent when the series is sorted by crescent intensities, as seen in Fig. 2(b). In other words, to build Fig. 2(b) we took the data of Fig. 2(a) and rearranged in crescent order from the minimum to the maximum intensity values. In Fig. 2(b) a remarkable change in the pattern of output intensities separating the two types of emission can be clearly observed. Indeed, in Fig. 2(b) and inset of Fig. 2(c) the spontaneous emission events shown in green can be readily distinguished from the RL intensity peak values depicted in blue. Based on this procedure, the weights $p$ and $1-p$ of each distribution were determined. Figure 2(c) displays in solid red line the nice fit to the statistical mixture, with the lowI gamma (high-I Lévy) component shown in dashed green (blue) line.

We comment that statistical mixtures have also been reported in other photonic experiments with random fiber laser
(RFL) [49-53], and they may be eventually present as well in RLs based on dye solutions and $\mathrm{TiO}_{2}$ particles [48,54].

\section{CONTRIBUTION OF THE EFFECTIVE FIFTH-ORDER SUSCEPTIBILITY}

The set of measurements reported in this work also allowed to identify for an excitation power well above threshold $P / P_{t h}=3.9$ significant deviations from the statistical mixture near the threshold discussed in the previous section.

In order to describe these deviations, an extension of the previously presented effective model was developed which also takes into account the effects of higher-order nonlinearities, in particular the fifth-order susceptibility $\chi^{(5)}$. The respective contribution is considered in $\Theta^{(5)}\left(\chi^{(1)}, \chi^{(3)}, \chi^{(5)}\right)$ by adding $\frac{1}{(3 !)^{2}} \sum_{\text {spqrtu }} g_{\text {spqrtu }}^{(6)} a_{s} a_{p} a_{q} a_{r}^{*} a_{t}^{*} a_{u}^{*}$ to $\Theta^{(3)}\left(\chi^{(1)}, \chi^{(3)}\right)$. In this case, the associated Langevin equation reads as

$$
\frac{d I_{m}}{d t}=\bar{d}_{m} I_{m}-\bar{b}_{m} I_{m}^{2}-\bar{c}_{m} I_{m}^{3}+2 I_{m} \eta_{m}^{(1)},
$$

where $\bar{d}_{m}\left(\bar{b}_{m}\right)$ is associated with $g^{(2)}, g^{(4)}$, and $g^{(6)}\left(g^{(4)}\right.$ and $\left.g^{(6)}\right)$. The coefficient $\bar{c}_{m}$, related only to $g^{(6)}$, emerges as an exclusive contribution from the effective fifth-order susceptibility $\chi^{(5)}$, and modifies the stationary solution (5) of the associated Fokker-Plack equation to the form

$$
P\left(I_{m}\right)=\frac{1}{G_{m}} I_{m}^{\zeta_{m}-1} \exp \left(-\rho_{m} I_{m}^{2}-v_{m} I_{m}\right),
$$

where the free parameters are given by $\zeta_{m}=\bar{d}_{m} / 4 Q-$ $1, \rho_{m}=\bar{c}_{m} / Q$, and $v_{m}=\bar{b}_{m} / 4 Q$, with the normalization constant

$$
G_{m}=\left(2 \rho_{m}\right)^{-\zeta_{m} / 2} \Gamma\left(\zeta_{m}\right) \exp \left(\frac{v_{m}^{2}}{8 \rho_{m}}\right) D_{-\zeta_{m}}\left(\frac{v_{m}}{\sqrt{2 \rho_{m}}}\right),
$$

and $D_{\lambda}(x)$ representing the parabolic cylinder function [55]. The stationary solution of the model, Eq. (11), synthesizes, through its deviations from the statistical properties discussed in the previous section, the way that fifth-order nonlinear effects modify the RL emission spectra.

Interestingly, by using a different approach Izrailev introduced in [56] a distribution similar to Eq. (11) in a quite distinct context, in order to characterize the eigenvalues spectrum of classical and quantum systems with mixed dynamics, in which regular and chaotic behaviors may coexist [57]. As a matter of fact, the nearest-neighbor spacing distribution (NNSD) of quantum systems with mixed dynamics interpolates between the Poisson distribution of the regular regime and the Wigner-Dyson distribution (a type of Rayleigh distribution) in the chaotic regime [57,58]. Nevertheless, aside from the peculiar NNSD, quantum systems with mixed dynamics also exhibit other important characteristics not present in photonic RL systems, such as a power spectral density in the power-law form $f^{-\alpha}$, with the spectral exponent in the range $1 \leqslant \alpha \leqslant 2$ [59]. Furthermore, the spectral rigidity, measured by the Dyson-Mehta $\Delta_{3}(L)$ statistics, interpolates between $\Delta_{3}(L)=L / 15$ for the regular regime and $\Delta_{3}(L) \sim \pi^{-2} \ln L$ for the chaotic regime. Therefore, although the Izrailev distribution (11) might suggest a link between the PDF of emission intensities in RLs and the NNSD of quantum systems with 

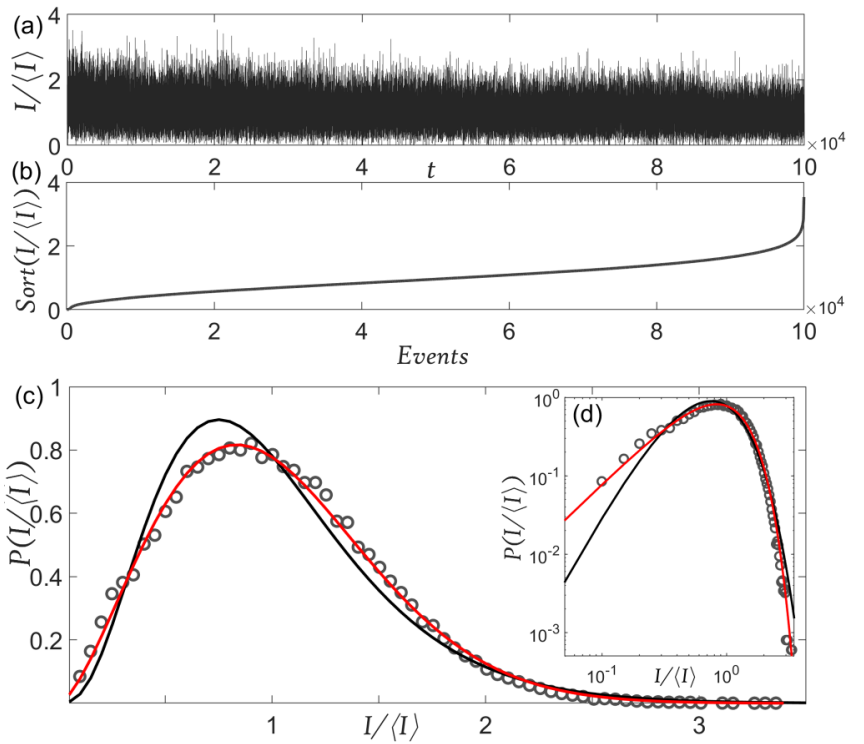

FIG. 3. (a) Long time series of emission intensities $I(t)$ at or very near the maximum of each spectrum (normalized by the mean $\langle I\rangle$ ) for an excitation power well above the RL threshold $P / P_{t h}=3.9$. (b) Sorting the data in (b) by crescent intensities, the abrupt change in Fig. 2(b) near the threshold is not observed. (c), (d) Linear and $\log -\log$ plots of the experimental distribution $P(I /\langle I\rangle)$ (circles), displaying a nice fit to the Izrailev PDF (11) (red lines) that takes into account nonlinear optical effects up to fifth order, with best-fit parameters $\zeta=2.6, \rho=0.77$, and $v=0.60$. The statistical mixture of Fig. 2(c) near the threshold no longer applies well above threshold since the contribution of the amplified spontaneous emission is negligible in this regime. Unsuccessful attempts to fit the data are also shown using the gamma distribution (5) (black lines) with $k=4.0$ and $b=4.0$, which incorporates nonlinear effects only up to third order.

mixed dynamics, we note that such connection in fact does not represent a complete analog map between these systems.

The influence of the fifth-order nonlinear optical effects on the statistics of intensity fluctuations can be directly inferred from the experimental data in the regime well above the RL threshold. Figure 3(a) shows the intensity time series for the normalized excitation power $P / P_{t h}=3.9$, while in Fig. 3(b) the data are sorted by crescent intensity values. Remarkably, the abrupt change observed in Fig. 2(b) near the threshold is no longer seen in Fig. 3(b) for $P / P_{t h}=3.9$. This result confirms that in the regime well above threshold the contribution from the amplified spontaneous emission is negligible, so that the statistical mixture of PDFs is no longer justified. Figures 3(c) and 3(d) display the nice fit of the experimental data of normalized intensities (circles) to the Izrailev distribution (red lines) [Eq. (11)]. Unsuccessful attempts to fit the experimental data are also shown using the PDF (5) (black lines) that incorporates nonlinear effects only up to third order. At this point, we comment that the positive best-fit value $\zeta=2.6$ of the Izrailev PDF (11) is also consistent with the Gaussian-type statistics of intensity fluctuations observed well above threshold in RL systems [21-26].

Therefore, we conclude that our model for the RL emission intensity distributions describes nicely the whole histogram of emission intensity values of the $\mathrm{Nd}^{3+}$-based $\mathrm{RL}$ system near and well above the laser threshold.

\section{FINAL REMARKS AND CONCLUSIONS}

In this work, measurements producing a very large and robust set of experimental data $\left(10^{5}\right.$ emission spectra) were used to characterize the distribution of intensities emitted by the disordered $\mathrm{Nd}^{3+}$-based nanocrystalline powder random laser. The statistical analysis of the histogram of emission peak intensities was done focusing on the regimes near and well above the lasing threshold, in which the effects of the third- and fifth-order nonlinearities are predominant.

For the lasing regime near the threshold, a statistical mixture of gamma and Lévy distributions indicates that the response of the random laser system varies from amplified spontaneous emission events to random lasing peaks. Based on the experimental evidence, it was shown that it suffices to take into account the influence of the third-order optical nonlinearity to characterize well the distribution of emission intensities close to the lasing threshold.

On the other hand, in the regime well above threshold the statistical mixture is not observed since the intensity emission spectra only incorporate laser peaks. In order to suitably describe this regime, a theoretical effective model was developed which takes into account the effects of the fifth-order nonlinearity, expressing the distribution of emission intensities in terms of the Izrailev distribution, with a nice fit to the experimental data. The Izrailev distribution is also found in the context of quantum systems with mixed chaotic and regular dynamics. We comment that our approach provides a dynamical model that explains the emergence of the Izrailev distribution.

Finally, we hope that this work can stimulate further experimental and theoretical studies in disordered random laser systems, especially above the lasing threshold in which highorder nonlinearity effects play an essential role.

\section{ACKNOWLEDGMENTS}

This work was supported by the Brazilian agencies Fundação de Amparo a Ciência e Tecnologia do Estado de Pernambuco (FACEPE), Coordenação de Aperfeiçoamento de Pessoal de Nível Superior (CAPES), and Conselho Nacional de Desenvolvimento Científico e Tecnológico (CNPq). The work was performed in the framework of the National Institute of Photonics (INCT de Fotônica-INFo) project. We gratefully acknowledge Dr. L. J. Q. Maia from Universidade Federal de Goiás for providing us the samples used in this work. E.P.R. would like to thank F. Bartumeus and the hospitality of the Spanish institutions.
[1] N. M. Lawandy, R. M. Balachandran, A. S. L. Gomes, and E. Sauvain, Nature (London) 368, 436 (1994).
[2] M. A. Noginov, Solid-State Random Lasers (Springer, Berlin, 2005). 
[3] R. Sapienza, Nat. Rev. Phys. 1, 690 (2019).

[4] H. Cao, J. Phys. A: Math. Gen. 38, 10497 (2005).

[5] F. Luan, B. Gu, A. S. L. Gomes, K.-T. Yong, S. Wen, and P. N. Prasad, Nano Today 10, 168 (2015).

[6] D. S. Wiersma, Nat. Phys. 4, 359 (2008).

[7] I. Viola, L. Leuzzi, C. Conti, and N. Ghofraniha, Basic physics and recent developments of organic random lasers, in Organic Lasers, edited by M. Anni and S. Lattante (Jenny Stanford Publishing, New York, 2018), Chap. 4.

[8] R. C. Polson and Z. V. Vardeny, Appl. Phys. Lett. 85, 1289 (2004).

[9] R. C. Polson and Z. V. Vardeny, J. Opt. 12, 024010 (2010).

[10] Y. Wang, Z. Duan, Z. Qiu, P. Zhang, J. Wu, D. Zhang, and T. Xiang, Sci. Rep. 7, 8385 (2017).

[11] I. Iparraguirre, J. Azkargorta, J. Fernández, R. Balda, S. GarcíaRevilla, and N. Hakmeh, Opt. Lett. 38, 3646 (2013).

[12] S. J. M. Carreño, A. L. Moura, P. I. R. Pincheira, Z. V. Fabris, L. J. Q. Maia, A. S. L. Gomes, and C. B. de Araújo, Opt. Mater. 54, 262 (2016).

[13] J. Azkargorta, I. Iparraguirre, J. Fernández, R. Balda, S. GarcíaRevilla, and M. Barredo-Zurriarrain, Opt. Express 26, 11787 (2018).

[14] P. K. Shahi, P. Singh, S. B. Rai, and A. Bahadur, Inorg. Chem. 55, 1535 (2016).

[15] M. Barredo-Zuriarrain, I. Iparraguirre, J. Fernández, J. Azkargorta, and R. Balda, Laser Phys. Lett. 14, 106201 (2017).

[16] A. L. Moura, P. I. R. Pincheira, L. J. Q. Maia, A. S. L. Gomes, and C. B. de Araújo, J. Lumin. 181, 44 (2017).

[17] A. L. Moura, V. Jerez, L. J. Q. Maia, A. S. L. Gomes, and C. B. de Araújo, Sci. Rep. 5, 13816 (2015).

[18] A. L. Moura, S. J. Carreño, P. I. R. Pincheira, L. J. Q. Maia, V. Jerez, E. P. Raposo, A. S. L. Gomes, and C. B. de Araújo, Appl. Opt. 59, D155 (2020).

[19] A. S. L. Gomes, E. P. Raposo, A. L. Moura, S. I. Fewo, P. I. R. Pincheira, V. Jerez, L. J. Q. Maia, and C. B. de Araújo, Sci. Rep. 6, 27987 (2016).

[20] E. P. Raposo and A. S. L. Gomes, Phys. Rev. A 91, 043827 (2015).

[21] R. Uppu and S. Mujumdar, Phys. Rev. Lett. 114, 183903 (2015).

[22] R. Uppu and S. Mujumdar, Phys. Rev. A 90, 025801 (2014).

[23] R. Uppu and S. Mujumdar, Phys. Rev. A 87, 013822 (2013).

[24] R. Uppu, A. K. Tiwari, and S. Mujumdar, Opt. Lett. 37, 662 (2012).

[25] R. Uppu and S. Mujumdar, Appl. Opt. 50, E13 (2011).

[26] R. Uppu and S. Mujumdar, Opt. Lett. 35, 2831 (2010).

[27] P. N. Butcher and D. Cotter, The Elements of Nonlinear Optics (Cambridge University Press, Cambridge, 1990).

[28] L. Angelani, C. Conti, G. Ruocco, and F. Zamponi, Phys. Rev. Lett. 96, 065702 (2006).

[29] L. Angelani, C. Conti, G. Ruocco, and F. Zamponi, Phys. Rev. B 74, 104207 (2006).

[30] F. Antenucci, C. Conti, A. Crisanti, and L. Leuzzi, Phys. Rev. Lett. 114, 043901 (2015).

[31] F. Antenucci, A. Crisanti, and L. Leuzzi, Phys. Rev. A 91, 053816 (2015).

[32] A. Schenzle and H. Brand, Phys. Rev. A 20, 1628 (1979).

[33] H. Haken, Light (North-Holland, Amsterdam, 1985), Vol. 2.

[34] V. M. Zolotarev and V. M. Uchaikin, Chance and Stability:
Stable Distributions and their Applications (De Gruyter, Utrecht, 1999).

[35] S. V. Buldyrev, A. L. Goldberger, S. Havlin, C.-K. Peng, M. Simons, and H. E. Stanley, Phys. Rev. E 47, 4514 (1993).

[36] S. Lepri, Phys. Rev. Lett. 110, 230603 (2013).

[37] S. Lepri, S. Cavalieri, G.-L. Oppo, and D. S. Wiersma, Phys. Rev. A 75, 063820 (2007).

[38] R. N. Mantegna and H. E. Stanley, Phys. Rev. Lett. 73, 2946 (1994).

[39] I. Koponen, Phys. Rev. E 52, 1197 (1995).

[40] F. Bartumeus, E. P. Raposo, G. M. Viswanathan, and M. G. E. da Luz, PLoS ONE 9, e106373 (2014).

[41] D. Anglos, A. Stassinopoulos, R. N. Das, G. Zacharakis, M. Psyllaki, R. Jakubiak, R. A. Vaia, E. P. Giannelis, and S. H. Anastasiadis, J. Opt. Soc. Am. B 21, 208 (2004).

[42] D. Sharma, H. Ramachandran, and N. Kumar, Opt. Lett. 31, 1806 (2006).

[43] K. L. van der Molen, A. P. Mosk, and A. Lagendijk, Phys. Rev. A 74, 053808 (2006).

[44] S. Mujumdar, V. Turck, R. Torre, and D. S. Wiersma, Phys. Rev. A 76, 033807 (2007).

[45] C. B. de Araújo, A. S. L. Gomes, and E. P. Raposo, Appl. Sci. 7, 644 (2017).

[46] B. C. Lima, P. I. R. Pincheira, E. P. Raposo, L. de S. Menezes, C. B. de Araújo, A. S. L. Gomes, and R. Kashyap, Phys. Rev. A 96, 013834 (2017).

[47] J. Li, H. Wu, Z. Wang, S. Lin, C. Lu, E. P. Raposo, A. S. L. Gomes, and Y. Rao, Opt. Lett. 44, 2799 (2019).

[48] X. Wu and H. Cao, Phys. Rev. A. 77, 013832 (2008).

[49] I. R. R. González, B. C. Lima, P. I. R. Pincheira, A. A. Brum, A. M. S. Macêdo, G. L. Vasconcelos, L. de S. Menezes, E. P. Raposo, A. S. L. Gomes, and R. Kashyap, Nat. Commun. 8, 15731 (2017).

[50] I. R. R. González, E. P. Raposo, A. M. S. Macêdo, L. de S. Menezes, and A. S. L. Gomes, Sci. Rep. 8, 17046 (2018).

[51] A. M. S. Macêdo, I. R. R. González, E. P. Raposo, L. de S. Menezes, and A. S. L. Gomes, Atoms 7, 43 (2019).

[52] B. C. Lima, A. S. L. Gomes, P. I. R. Pincheira, A. L. Moura, M. Gagné, E. P. Raposo, C. B. de Araújo, and R. Kashyap, J. Opt. Soc. Am. B 34, 293 (2017).

[53] E. P. Raposo, I. R. R. González, A. M. S. Macêdo, B. C. Lima, R. Kashyap, L. de S. Menezes, and A. S. L. Gomes, Phys. Rev. Lett. 122, 143903 (2019).

[54] P. I. R. Pincheira, A. F. Silva, S. J. M. Carreño, A. L. Moura, S. I. Fewo, E. P. Raposo, A. S. L. Gomes, and C. B. de Araújo, Opt. Lett. 41, 3459 (2016).

[55] H. Bateman and A. Erdélyi, Tables of Integral Transforms (McGraw-Hill, New York, 1954), Vol. 1.

[56] F. M. Izrailev, J. Phys. A: Math. Gen. 22, 865 (1989).

[57] A. Richter, Playing billiards with microwaves: Quantum manifestations of classical chaos, in Emerging Applications of Number Theory, edited by D. A. Hejhal, J. Friedman, M. C. Gutzwiller, and A. M. Odlyzko (Springer, New York, 1999), p. 479.

[58] M. L. Mehta, Random Matrices (Elsevier, San Diego, 2004).

[59] J. M. G. Gómez, A. Relaño, J. Retamosa, E. Faleiro, L. Salasnich, M. Vranicar, and M. Robnik, Phys. Rev. Lett. 94, 084101 (2005). 